Revista Destaques Acadêmicos, Lajeado, v. 10, n. 3, 2018. ISSN 2176-3070

DOI: http://dx.doi.org/10.22410/issn.2176-3070.v10i3a2018.1738

http://www.univates.br/revistas

\title{
QUALIDADE DE VIDA DE MULHERES COM DIFERENTES FAIXAS ETÁRIAS DE UM PROGRAMA DE PROMOÇÃO À SAÚDE
}

\author{
Kelly Cristina Lasta ${ }^{1}$, Luiz Gabriel da Silva ${ }^{2}$, Higor Rissini Ferrari ${ }^{3}$, \\ Jéssica Luana Dornelles da Costa ${ }^{4}$, Caroline Pieta Dias ${ }^{5}$, \\ Carlos Leandro Tiggemann ${ }^{6}$
}

\begin{abstract}
Resumo: O objetivo do estudo foi comparar a qualidade de vida de mulheres de diferentes faixas etárias que participam de um programa de promoção à saúde. A amostra foi constituída por 244 mulheres, divididas em três grupos: com idade até 59 anos (G1), de 60 a 69 anos (G2) e com 70 anos ou mais (G3). Para a coleta de dados foi utilizado questionário sociodemográfico e de qualidade de vida (SF-36). Os dados foram analisados por meio de análise descritiva, distribuição da frequência e comparação das médias pelo teste de Kruskal-Wallis $(\mathrm{p}<0,05)$. Somente foram encontradas diferenças significativas $(\mathrm{p}<0,05)$ nos domínios capacidade funcional e limitação por aspectos emocionais entre os grupos G1 e G3, sendo os melhores escores em favor as mulheres mais jovens. Os resultados permitem concluir que na maioria dos domínios as mulheres apresentam uma qualidade de vida similar, ou seja, diferentes faixas etárias não repercutem em mudanças na qualidade de vida.
\end{abstract}

Palavras-chave: Qualidade de vida. Mulheres. Faixas etárias.

1 Profissional de Educação Física Bacharelado - Univates - Lajeado/RS - kelly_lasta@yahoo.com.br

2 Graduando em Educação Física Bacharelado - Univates - Lajeado/RS - luiz.silva4@univates.br

3 Graduando em Educação Física Bacharelado - Univates - Lajeado/RS - higor.ferrari@univates.br

4 Graduada em Educação Física licenciatura - Univates - Lajeado/RS - jluana@univates.br

5 Doutora em Ciência do Movimento Humano - Universidade Federal do Rio Grande do Sul (UFRGS) - Porto Alegre/RS - carolpieta@yahoo.com.br

6 Doutor em Ciência do Movimento Humano - Universidade Federal do Rio Grande do Sul (UFRGS) - Porto Alegre/RS - cltiggemann@univates.br 


\section{INTRODUÇÃO}

A idade cronológica serve como um demarcador social para identificar o início da velhice, considerando idoso todo sujeito com 60 anos ou mais (BLESSMANN, 2003). Sabe-se que o envelhecimento populacional vem crescendo nos últimos anos, devido às mudanças do quadro demográfico. $\mathrm{O}$ número de pessoas com mais de 60 anos está aumentando drasticamente, com uma estimativa para 2025 de 1,2 bilhões de pessoas e em 2050 este número pode chegar a 2 bilhões, ocorrendo principalmente em países em processo de desenvolvimento (OMS, 2015).

$\mathrm{O}$ processo do envelhecimento deve ser considerado dinâmico e progressivo, onde ao avançar da vida ocorrem modificações no sistema biológico, psíquico e social (MINCATO, 2007). Alterações estruturais e funcionais de órgãos e tecidos comprometem a capacidade física do indivíduo idoso, e essas alterações é de individualidade de cada pessoa e não apenas da faixa etária (JUNIOR, 2009; MATSUDO, 1992). Também o equilíbrio social é afetado, pois o isolamento e esquecimento dos idosos pode prejudicar o psicológico, levando à baixa auto-estima, crises de ansiedade e depressão (CASAGRANDE, 2006).

Neste sentido, para o indivíduo envelhecer saudável, deve incluir boa saúde física e mental, ser independente, ter um convívio social, boa estrutura familiar e situação financeira (RAMOS, 2003). Com a prática de atividade física os efeitos do envelhecimento podem ser minimizados, melhorando a força, a massa muscular, a flexibilidade, o sistema circulatório, o aumento da capacidade cardiorrespiratória, a glicemia controlada e um melhor equilíbrio, diminuindo a incidência de quedas, além da melhora da auto-estima e autoconfiança (NOBREGA, 1999).

O conceito de qualidade de vida é um termo complexo e está relacionado com a satisfação de cada indivíduo em relação às dimensões da família, sociedade, auto imagem, estado emocional e bem-estar. Pode ser entendido como um conceito subjetivo que irá variar do nível sociocultural, do perfil etário e dos desejos de cada pessoa (MINAYO, 2000; VECCHIA, 2005). A satisfação consigo mesmo e o bem-estar geral podem ser vistos como uma grandeza particular, complexa e multidimensional. Entender e conhecer estes fatores que são influenciados diretamente pela saúde, idade cronológica, renda, raça, emprego, estado civil, locomoção, moradia, atividade com a sociedade, é de fundamental importância (OKUMA, 2012).

Uma das formas para contribuir com a qualidade de vida é a participação das pessoas em programas sociais, realizados nos diferentes municípios, os quais têm o propósito de intervir através de programas de exercícios físicos e atividades socioeducativas (SILVA, 2011; SILVESTRE, 2003). A atividade física pode beneficiar a saúde física, fisiológica, diminuir os declínios funcionais, melhorar a capacidade motora e a autonomia e também, servir como um 
instrumento para equilibrar os domínios psíquicos, sociais e ser essencial na promoção da saúde e da qualidade de vida (NOBREGA, 1999; TOSCANO, 2009; VICCHIA, 2005).

Neste contexto, o estudo de Mota et al. (2006) teve por objetivo avaliar o impacto da atividade física na qualidade de vida em participantes e não participantes de um programa de atividade física, sendo que participaram 88 sujeitos de ambos os sexos, com mais de 65 anos. As atividades proporcionadas foram ritmos, caminhadas, exercícios de força e de resistência, entre outras. Os autores comprovaram em seu estudo que os idosos, ao participarem do programa de atividade física, tiveram um nível de qualidade de vida mais elevado do que aqueles que não participaram, concluindo que a participação em programas de promoção à saúde melhora a qualidade de vida.

Um dos aspectos que é pouco investigado na literatura, diz respeito à qualidade de vida em diferentes estratos etários. Neste sentido, uma pesquisa que avaliou a qualidade de vida comparando a faixa etária de idosos jovens de 60 a 70 anos e idosos com idade mais avançada, 85 anos ou mais, observou que a qualidade de vida foi considerada boa e que não piora com o avanço da idade (MAUÉS, 2010). Desta forma, percebendo a carência de estudos acerca deste tema, o presente estudo teve como objetivo avaliar e comparar a qualidade de vida em mulheres de diferentes faixas etárias que participam de um programa de promoção à saúde.

\section{MÉTODO}

Trata-se de uma pesquisa de caráter quantitativo, descritivo e expost facto. Todos os procedimentos de coleta iniciaram logo após a aprovação pelo Comitê de Etica do Centro Universitário Univates (parecer $\mathrm{n}^{\circ} 1.349 .615$ ), sendo que todos os sujeitos assinaram o Termo de Consentimento Livre e Esclarecido. A amostra foi constituída por 244 mulheres com idade entre 35 a 85 anos, divididas em três grupos: com idade até 59 anos $(\mathrm{G} 1, \mathrm{n}=81)$, de 60 a 69 anos (G2, n=116) e com 70 anos ou mais (G3, n= 47). Estes sujeitos advêm de 15 dos 17 grupos de um programa de promoção à saúde do Município de Encantado-RS. Para participar do estudo, os sujeitos deveriam estar residindo no referido município, possuir idade a partir de 35 anos, ser do sexo feminino e estar frequentando e participando das atividades oferecidas pelo programa regularmente. Os dados de caracterização dos sujeitos estão apresentados na tabela 1.

Para a coleta dos dados foram utilizados dois questionários. Um constituído de questões relacionadas às informações sociodemográficas e de características físicas, e outro, o questionário de qualidade de vida SF-36 (Medical Out Comes Study 36 - Item Short-From Health Survey). O mesmo tem por finalidade obter informações referentes a qualidade de vida, contendo 36 questões, subdivididas em oito domínios (capacidade funcional, aspectos 
físicos, dor, estado geral de saúde, vitalidade, aspectos sociais, aspectos emocionais e saúde mental). Para o tratamento das respostas, foram utilizados os critérios do instrumento de avaliação, sendo que os escores variam de 0 a 100, onde o escore 0 classifica o sujeito em pior estado de saúde e escore 100 sujeitos com um melhor estado de saúde (CICONELLI, 1999). Ambos os questionários foram aplicados no mês de março de 2016, nos locais em que os sujeitos realizavam as atividades físicas, no período da tarde e no horário em que aconteciam as sessões. A coleta foi realizada em pequenos grupos, sendo que para os sujeitos analfabetos, os dados foram adquiridos por meio de leitura de forma individualizada.

Tabela 1 - Variáveis de caracterização sociodemográfica da amostra $(n=244)$

\begin{tabular}{l|c|c}
\hline Características & $\mathbf{n}$ & $\%$ \\
\hline Etnia & 230 & 94,3 \\
\hline Branca & 3 & 1,2 \\
\hline Negra & 11 & 4,5 \\
\hline Parda & & \\
\hline Estado Civil & 18 & 7,4 \\
\hline Solteiro/Separado/Divorciado & 168 & 68,9 \\
\hline Casado & 58 & 23,8 \\
\hline Viúvo & & \\
\hline Renda & 23 & 9,4 \\
\hline Sem renda & 189 & 77,5 \\
\hline Até 2 salários mínimos & 32 & 13,1 \\
\hline Mais que 2 salários mínimos & 90 & 36,9 \\
\hline Local Zona & 154 & 63,1 \\
\hline Zona Rural & & \\
\hline Zona Urbana & 151 & 61,9 \\
\hline Escolaridade & 93 & 38,1 \\
\hline Sem escolaridade/ $1^{\circ}$ grau incompleto & & \\
\hline $1^{\circ}$ grau completo ou mais & 53 & 21,7 \\
\hline Tempo de participação no grupo & 33 & 13,5 \\
\hline Até 1 ano & 40 & 16,4 \\
\hline De 1 até 2 anos & 118 & 48,4 \\
\hline De 2 até 3 anos & & \\
\hline Mais de 3 anos & & \\
\hline
\end{tabular}


A análise estatística dos dados foi realizada por meio da análise descritiva com média, desvio padrão e distribuição da frequência. Por meio do teste de Shapiro-Wilk, constatou-se que os dados não se enquadraram em um padrão de normalidade e, para tanto, utilizou-se o teste não paramétrico de Kruskal-Wallis, a fim comparar as médias dos domínios de qualidade de vida. Todos os procedimentos foram realizados no programa estatístico, software SPSS v. 18.0, com nível de significância $\mathrm{p}<0,05$.

\section{RESULTADOS}

Na Tabela 2 estão representados as médias e desvios padrões das idades e dos domínios da qualidade de vida, na amostra geral e estratificada pelos subgrupos quanto à idade. Foram observadas diferenças significativas em relação à idade entre todos os grupos $(p<0,001)$. Em relação à qualidade de vida, houve diferença significativa nos domínios da capacidade funcional e limitação por aspectos emocionais entre o G1 e G3, sendo que, os melhores escores foram de mulheres mais jovens comparadas com aquelas com idade mais avançada. Não foram encontradas diferenças entre os grupos nos demais domínios de qualidade de vida.

Tabela 2 - Médias e desvios padrões da idade e domínios da qualidade de vida da amostra geral e subgrupos.

\begin{tabular}{l|c|c|c|c}
\hline \multicolumn{1}{c|}{ Variável } & Geral & G1 & G2 & G3 \\
\hline Idade & $62,1 \pm 9,6$ & $51,4 \pm 6,4^{\mathrm{a}}$ & $64,3 \pm 2,5^{\mathrm{b}}$ & $74,9 \pm 4,5^{\mathrm{c}}$ \\
\hline Capacidade Funcional & $82,3 \pm 15,3$ & $85,9 \pm 13,0^{\mathrm{a}}$ & $81,3 \pm 16,0^{\mathrm{ab}}$ & $78,4 \pm 16,2^{\mathrm{b}}$ \\
\hline Limitação por Aspectos Físicos & $79,0 \pm 32,4$ & $80,9 \pm 31,4^{\mathrm{a}}$ & $80,0 \pm 32,2^{\mathrm{a}}$ & $73,4 \pm 34,7^{\mathrm{a}}$ \\
\hline Dor & $66,2 \pm 18,6$ & $65,9 \pm 19,4^{\mathrm{a}}$ & $67,2 \pm 18,9^{\mathrm{a}}$ & $64,3 \pm 16,8^{\mathrm{a}}$ \\
\hline Estado Geral de Saúde & $60,2 \pm 12,8$ & $59,4 \pm 13,7^{\mathrm{a}}$ & $60,4 \pm 12,2^{\mathrm{a}}$ & $61,2 \pm 12,7^{\mathrm{a}}$ \\
\hline Vitalidade & $67,4 \pm 14,6$ & $67,5 \pm 13,1^{\mathrm{a}}$ & $68,5 \pm 14,2^{\mathrm{a}}$ & $64,5 \pm 17,9^{\mathrm{a}}$ \\
\hline Aspectos Sociais & $82,8 \pm 16,6$ & $85,0 \pm 15,5^{\mathrm{a}}$ & $81,5 \pm 17,3^{\mathrm{a}}$ & $84,1 \pm 16,4^{\mathrm{a}}$ \\
\hline Limitação Aspectos Emocionais & $80,9 \pm 32,0$ & $85,6 \pm 27,9^{\mathrm{a}}$ & $81,0 \pm 32,7^{\mathrm{ab}}$ & $72,3 \pm 35,7^{\mathrm{b}}$ \\
\hline Saúde Mental & $71,6 \pm 14,0$ & $70,5 \pm 13,2^{\mathrm{a}}$ & $71,6 \pm 14,5^{\mathrm{a}}$ & $73,4 \pm 14,3^{\mathrm{a}}$ \\
\hline
\end{tabular}

Legenda: Geral ( $\mathrm{n}=244) ; \mathrm{G1}$ (até 59 anos; $\mathrm{n}=81)$; G2 (60-69 anos; $\mathrm{n}=116) ; \mathrm{G} 3$ (>69 anos; $\mathrm{n}=47)$. Letras diferentes indicam diferenças significativas entre os grupos $(\mathrm{p}<0,05)$.

\section{DISCUSSÃO}

O objetivo deste estudo foi avaliar a qualidade de vida em mulheres de diferentes faixas etárias que participam de um programa de promoção à saúde. Nas verificações quanto aos escores gerais da qualidade de vida de todas 
as mulheres, um melhor valor foi encontrado nos domínios de capacidade funcional $(82,3 \pm 15,3)$, aspectos sociais $(82,8 \pm 16,6)$ e limitações por aspectos emocionais $(80,9 \pm 32,0)$. Como hipótese, acreditamos que possivelmente o convívio social nos grupos possa ser um dos responsáveis por trazer benefícios para as pessoas, como a autonomia, interação no meio social e bem-estar (LEITE, 2012).

Outro fator que pode ter contribuído, diz respeito aos conteúdos dos programas de promoção à saúde, no caso, a prática de atividades físicas. $\mathrm{O}$ estudo de Toscano e Oliveira (2009) afirma que programas de atividade física são indispensáveis e contribuem na qualidade de vida do sujeito idoso, desde envolvimento social até na questão física, resultando em maior autonomia. Igualmente, Gonçalves et al. (2011) tinham por objetivo avaliar o impacto da atividade física na qualidade de vida das mulheres de meia idade, sendo constatado que a mesma é fundamental para a qualidade de vida. Outros autores afirmam que programas de atividade física auxiliam na manutenção dos componentes de aptidão funcional, melhoram a coordenação, flexibilidade, agilidade, equilíbrio, resistência de força e aeróbica, sendo fundamentais para um envelhecimento com saúde e qualidade (MATSUDO, 2009; PAULI, 2009).

Em nosso estudo, também observamos que alguns domínios obtiveram escores inferiores, como o que se refere a dor $(66,2 \pm 18,6)$, estado geral de saúde $(60,2 \pm 12,8)$ e vitalidade $(67,4 \pm 14,6)$. Estes resultados são semelhantes aos da pesquisa de Leite et al. (2012), que realizaram um estudo em um município do Rio Grande do Sul, com o objetivo de avaliar a qualidade de vida e o nível cognitivo de idosos participantes de grupos de terceira idade, indicando piores valores nos domínios da dor, vitalidade e estado geral de saúde. Uma possível explicação para estes resultados seria o processo de envelhecimento, o qual produz alterações físicas, psicológicas e sociais, as quais acometem o sujeito idoso provocando um decréscimo na capacidade funcional. Além disso, o decréscimo nos fatores sócio-culturais e ambientais podem acarretar a esse público um baixo nível da sua capacidade, em conseqüência disso, aparecem problemas de saúde e tornando-o dependente de outra pessoa (SILVA, 2011).

Quando comparados os diferentes estratos etários, o presente estudo constata que o domínio de capacidade funcional no grupo mais velho (G3) é significativamente inferior quando se comparado ao grupo mais jovem (G1), mostrando que as mulheres de até 59 anos apresentam uma melhor capacidade funcional do que aquelas com 70 anos ou mais. Estes resultados corroboram com os achados de Pereira, Cardoso e Machado (2011), que avaliaram o perfil da força de preensão de mulheres em diferentes faixas etárias $(20-27,50-64,65-$ 74 e 75-86 anos), sendo identificado um menor valor da força em idosas de 75-86 anos, comparando com as de 50-64 e 65-74 anos. Já é evidenciado na literatura o declínio funcional exponencial que acontece, junto com a diminuição da massa muscular, a cada década de idade (NOBREGA, 1999; RIKLES; JONES, 1999; DEL, 2009; KAUFMAN, 2012). 
Com o avançar da idade, além das alterações estruturais, ocorre o declínio das funções psicológicas (OKUMA, 2012). Neste estudo, quanto ao domínio das limitações por aspectos emocionais, foi constatado um escore superior no grupo mais jovem (G1) quando comparado com o grupo mais velho (G3). Uma hipótese que possa explicar esse resultado estaria na dificuldade dos sujeitos mais velhos em aceitar a velhice. Com a chegada da terceira idade e a aposentadoria, com a diminuição das atividades da vida diária que são desempenhadas dentro do lar e com as mudanças físicas que são visíveis nessa fase, ocorre a diminuição de rendimento e da socialização, muitas vezes perdendo o poder de decisão em assuntos de família (FILHO, 1999). Devido a estes fatores, pode ocorrer a infelicidade, o isolamento e a depressão, que comprometem a estrutura psicológica da pessoa (DUARTE, 2002).

Uma das atividades que se destaca dentro de programas sociais tem relação com a dança. Oliveira, Pivoto e Vianna (2009) que avaliaram o impacto da dança sênior na saúde de 103 idosos, sendo observado que o domínio de aspecto emocional obteve o melhor resultado. Os autores ainda destacam a importância dessa atividade em outros aspectos, como a melhora na socialização, nos sentimentos de depressão, bem-estar, equilíbrio emocional, trazendo mais energia para os afazeres do dia a dia dessas pessoas. Ainda, em estudo de Brunoni et al. (2015), foi constatado que o treinamento de força foi eficaz para diminuir os sintomas da depressão e melhorar a qualidade de vida relacionada à saúde, nos domínios, vitalidade, capacidade funcional, estado geral de saúde e saúde mental.

Na pesquisa de Cavallini et al. (2012), foi comparada a qualidade de vida de idosos de diferentes faixas etárias (60 a 80 anos), integrantes dos projetos do município de Cruz Alta/RS. Os autores constataram que, idosos da faixa etária mais nova (60-64 anos) obtiveram escores similares em todos os domínios, concluindo que ambos apresentaram uma qualidade de vida similar. Desta forma, pode-se presumir que um dos possíveis fatores que possa estar ajudando na manutenção dessas variáveis, seja o convívio social entre as pessoas no programa de promoção à saúde.

Portanto, a avaliação e o monitoramento da qualidade de vida é um instrumento importante, pois auxilia o profissional da saúde a melhorar as suas intervenções e ações. Acredita-se também, que a participação das pessoas em programas ou projetos de promoção à saúde possa auxiliar em um envelhecimento saudável, um padrão de saúde melhor e uma boa qualidade de vida (FREITAS 2007; TOSCANO, 2009). Importante observar, que cada domínio tem sua importância na qualidade de vida, devendo a mesma ser entendida como um conceito subjetivo que variará dos desejos de cada pessoa (VECCHIA, 2005; NERI, 2012). 


\section{CONCLUSÃO}

Desta forma, pode-se concluir com este estudo que o envelhecimento não acarreta em uma redução no escore da maioria dos domínios da qualidade de vida em mulheres participantes de programas de promoção à saúde, sendo que somente os domínios da capacidade funcional e limitação por aspectos emocionais apresentaram menores escores na faixa etária mais velha.

\section{REFERÊNCIA}

BLESSMANN EJ. Corporeidade e envelhecimento: o significado do corpo na velhice. Dissertação (Mestrado). Universidade Federal do Rio Grande do Sul, Ciências do Movimento Humano; 2003.

BRUNONI, Letícia et al. Treinamento de força diminui os sintomas depressivos e melhora a qualidade de vida relacionada a saúde em idosas. Revista Brasileira de Educação Física e Esporte, v. 29, n. 2, p. 189-196, 2015.

CASAGRANDE M. Atividade física na terceira idade. Monografia (Graduação). Bauru: Faculdade de Ciências da Unesp, Curso de Licenciatura Plena em Educação Física; 2006.

CAVALLINI GM et al. Avaliação da qualidade de vida subjetiva dos idosos: uma comparação entre diferentes faixas etárias. XVII Seminário Interinstitucional de Ensino, Pesquisa e Extensão. Nov 6-8; Cruz Alta, RS. Brasil: UNICRUZ;2012.

CICONELLI, Rozana Mesquita. Traduçäo para o português e validaçäo do questionário genérico de avaliaçäo de qualidade de vida. Tese de Doutorado. Universidade Federal de Sao Paulo. Escola Paulista de Medicina; 1997.

DEL duca GF, Silva MC, Hallal PC. Incapacidade funcional para atividades básicas e instrumentais da vida diária em idosos. Revista de Saúde Pública.43(5):796-805;2009.

DUARTE CP, Santos CL, Gonçalves AK. A concepção de pessoas de meia-idade sobre saúde, envelhecimento e atividade física como motivação para comportamentos ativos. Revista Brasileira de Ciências do Esporte v. 23, n. 3, p. 35-48, 2002.

FILHO LFB. O processo do envelhecimento e o comportamento vocal. Monografia (Especialização). Curso de especialização em Voz, Centro de Especialização em Fonoaudiologia Clínica. 1999.

FREITAS CMSM et al. Aspectos motivacionais que influenciam a adesão e manutenção de idosos a programas de exercícios físicos. Revista Brasileira de cineantropometria e desempenho humano v. 9, n.1, p. 92-10, 2007.

GONÇALVES AKS. Impacto da atividade física na qualidade de vida de mulheres de meia idade: estudo de base populacional. Revista brasileira de ginecologia e obstetrícia, v. 33, n. 12, p. 408-13, 2011. 
JUNIOR, Edmundo. Envelhecimento e vida saudável. Apicuri, 2009.

KAUFMAN, Fani G. Novo velho: envelhecimento, olhares e perspectivas. São Paulo: Casa do Psicólogo, 2012.

LEITE, Marinês Tambara et al. Qualidade de vida e nível cognitivo de pessoas idosas participantes de grupos de convivência. Revista brasileira geriatria gerontologia, $\mathrm{v}$. 15, n. 3, p. 481-492, 2012.

MATSUDO, Sandra M.; MATSUDO, Victor KR. Prescrição e benefícios da atividade física na terceira idade. Revista Brasileira de Ciência e Movimento, v. 6, n. 4, p. 19-30, 2008.

MATSUDO SMM. Envelhecimento, atividade física e saúde. BIS. Boletim do Instituto de Saúde, v. 47, p.76-79, 2009.

MAUÉS CR. Avaliação da qualidade de vida: comparação entre idosos jovens e muito idosos. Revista Brasileira Clínica Médica, v.8, n. 5, p. 405-10, 2010.

MINAYO MCS, Hartz ZMA, Buss PM. Qualidade de vida e saúde: um debate necessário. Ciência da Saúde Coletiva, v.5, n. 1, p. 7-18, 2000.

MINCATO, Paula Cristina; FREITAS, Cíntia de La Rocha. Qualidade de vida dos idosos residentes em instituições asilares da cidade de Caxias do Sul-RS. Revista Brasileira de Ciências do Envelhecimento Humano, v. 4, n. 1, 2007.

MOTA, Jorge et al. Atividade física e qualidade de vida associada à saúde em idosos participantes e não participantes em programas regulares de atividade física. Revista brasileira de educação fìsica e esporte, v. 20, n. 3, p. 219-225, 2006.

NERI, Anita Liberalesso. Qualidade de vida e idade madura. Papirus editora, 1993.

NÓBREGA, Antonio Claudio Lucas da et al. Posicionamento oficial da Sociedade Brasileira de Medicina do Esporte e da Sociedade Brasileira de Geriatria e Gerontologia: atividade física e saúde no idoso. Revista brasileira de medicina do esporte, v. 5, n. 6, p. 207-211, 1999.

OKUMA, S. S. O idoso e a atividade física: Fundamentos e pesquisa. $3^{\text {a }}$ edição. 1998.

ORGANIZAÇÃO MUNDIAL DA SAÚDE. Envelhecimento ativo: uma política de saúde. Brasília Organização Pan-Americana da Saúde, 2005. Disponível em: <http:/ / bvsms.saude.gov.br/bvs/publicacoes/envelhecimento_ativo.pdf > Acesso em: 5 ago. 2015.

PAULI, José Rodrigo et al. Influência de 12 anos de prática de atividade física regular em programa supervisionado para idosos. Revista Brasileira de cineantropometria e desempenho humano, v. 11, n. 3, p. 255-60, 2009. 
PEREIRA, Rafael et al. Análise da força de preensão de mulheres idosas: estudo comparativo entre faixas etárias. Acta Médica Portuguesa, v. 24, n. 4, p. 521-6, 2011.

RAMOS, Luiz Roberto. Fatores determinantes do envelhecimento saudável em idosos residentes em centro urbano: Projeto Epidoso, São Paulo. Cadernos de Saúde Pública, 2003.

RIKLI, Roberta E.; JONES, C. Jessie. Functional fitness normative scores for community-residing older adults, ages 60-94. Journal of Aging and Physical Activity, v. 7, n. 2, p. 162-181, 1999.

SILVA PJP. A influência da actividade física na qualidade de vida do idoso. Dissertação (Mestrado) Instituto Politécnico de Viseu, Escola Superior de Saúde de Viseu. 2011.

SILVESTRE, Jorge Alexandre; DA COSTA NETO, Milton Menezes. Abordagem do idoso em programas. Cadernos de Saúde Pública, v. 19, n. 3, p. 839-847, 2003.

TOSCANO, José Jean de Oliveira; OLIVEIRA, Antônio César Cabral de. Qualidade de vida em idosos com distintos níveis de atividade física. Revista brasileira de medicina do esporte, v. 15, n. 3, p. 169-173, 2009..

VECCHIA, Roberta Dalla et al. Qualidade de vida na terceira idade: um conceito subjetivo. Revista brasileira de epidemiologia, p. 246-252, 2005. 